
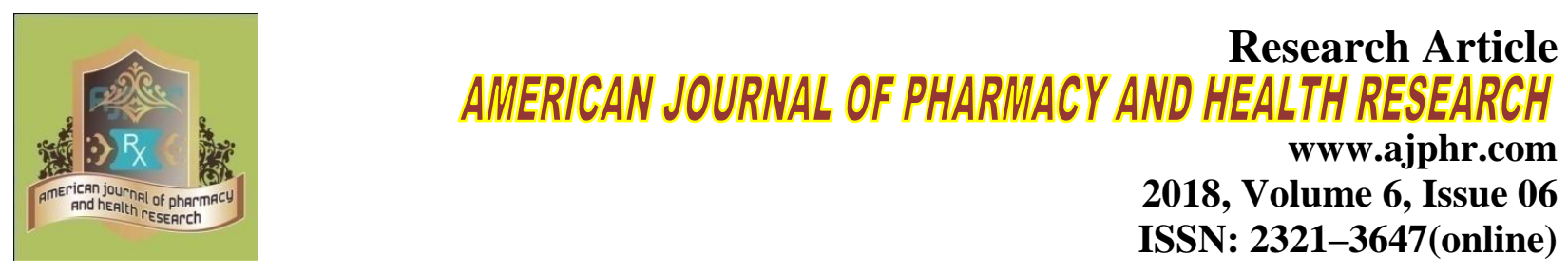

\title{
Formulation and Evaluation of Antimicrobial Activity of Topical Niosomal Gel of Erythromycin
}

\author{
Nettem Lalithajyotsna ${ }^{*}$, Mohammad Sarfaraz Ali Ahmed ${ }^{2}$, Mahendar \\ choudary ${ }^{3}$,Dr.vasudha Bakshi ${ }^{4}$ \\ Department of Biotechnology, Anurag Group of Institutions (Formerly Lalitha College of \\ Pharmacy) Venkatapur (v), Ranga Reddy-500088, Telangana, India.
}

\begin{abstract}
Erythromycin is a macrolide antibiotic produced by Streptomyces erythreus. It inhibits bacterial protein synthesis by binding to bacterial 50S ribosomal subunits; binding inhibits peptidyl transferase activity and interferes with translocation of amino acids during translation and assembly of proteins. Erythromycin may be bacteriostatic or bactericidal depending on the organism and drug concentration. The drugs in the form of topical gels avoid gastro-intestinal irritation, to overcome first pass effect and to maximize the drug concentration at the site of action. The main objective of the study is to formulate and evaluate the antimicrobial activity of erythromycin formulation for effective topical delivery of the drug. The antimicrobial activity of the erythromycin loaded gel is carried by cup-plate method and the zone of inhibition was noted. The current analysis demonstrates persistence release of drug, an increase in amount of drug maintenance into skin and enhanced permeation across the skin after entrapment of Erythromycin into niosomal topical gel.
\end{abstract}

Keywords: Niosomes, Erythromycin, Antimicrobial activity, topical delivery, cup-plate method, Niosomal Gel and Skin Retention.

*Corresponding Author Email: lalitha.jyotsna@gmail.com

Received 10 June 2018, Accepted 18 June 2018 


\section{INTRODUCTION}

Various Drug delivery systems by means of vesicles like liposomes and niosomes possess added recompense on the typical dosage forms. This is because the drug is entrapped in the vesicle and the vesicle act as a reservoir of the drugs. By changing the constitution of the vesicle and altering the attraction for the target site, the rate of release of the drug can monitor and slow down. This slow release of the drug can reduce the toxicity of the drug. Based on this principle these vehicle carriers' exhibit important functions in the delivery of the drugs. Niosomes and liposome are unilamellar or multilamellar vesicles in which an aqueous phase is encapsulated in substantially structured bilayer made up of nonionic surfactant (niosomes) or lipid (liposomes) with or without other components like, cholesterol (chol) and Dicetyl phosphate. $(1,2)$.

The two vesicular structures niosomes and liposomes show preferred interaction with human skin when applied through topical preparation. These drug dosage forms improve the horny layer character and decrease the loss of water through dermis and enhance the smoothness of skin by restocking skin lipids $(3,4)$. Both the vesicular structures posses more or less same advantage, but niosomes were Among niosomes and liposomes possess more or less same advantage, niosomes were considered to favorite vesicular carrier dosage forms. The drug encapsulated in niosomes exhibit contact with skin without exercising sudden or robust systemic action.(5,6,) The macrolide antibiotic Erythromycin can exhibit static and cidal action on bacteria based on the concentration of the dosage form and sensitivity of the pathogen.

Conventional topical usage of macrolide antibiotic causes undesirable side effects like irritation, eager, reddishness of skin etc., . This causes trouble in usage of the Erythromycin in the therapy and on the other hand development of antibiotic resistant bacteria.

In the present investigation the study was made based on the hypothesis that noisome encapsulation of macrolide antibiotic Erythromycin aid in increase in the quantity and time of drug retention within the skin. This enhances the therapeutic efficiency of the drug and reduction in its toxicity.

\section{MATERIALS AND METHOD}

\section{Materials}

Erythromycin, Span 80, Cholesterol, Chloroform, Carbopol 934, Propylene Glycol and ethanol were procured from UV Suppliers. The phosphate Buffer e of $\mathrm{pH} 7.4[\mathrm{PBS} \mathrm{pH} 7.4]$ and Phosphate Buffer Saline pH 6.8 [PBS pH 6.8] were made as mentioned in Indian Pharmacopoeia and necessary chemical were obtained from UV Suppliers. All the chemicals used were of 
Analytical Reagent (AR) grade unless otherwise specified and the HiMEDIA Erythromycin was as marketed preparation used.

\section{Formulation Erythromycin loaded Niosomes:}

Based on the literature survey the niosomes formulation was made and characterized .For the preparation of the niosomes 1:1 ratio of chloroform and methanol along with cholesterol, drug and span 80 were used. (Table-1) This mixture was uniformly stirred for proper mixing of the ingredients. The solution was rotated at $60^{\circ} \mathrm{c}$ in rota evaporator Sonics 569-00050-000 at 100 rpm for formation of thin film in the round bottomed flask.(Figure-1-A,B) The thin film was hydrated with PBS 7.4 and centrifuged for removal of free drug; the niosomes formulation was characterized by FTIR analytical studies. (7)

Table 1: Quantity of substances taken for preparation of niosomes

\begin{tabular}{llll}
\hline Formulation & Drug & Cholesterol & Span 80 \\
\hline F1 & $0.1 \mathrm{~g}$ & $0.1 \mathrm{~g}$ & $0.1 \mathrm{ml}$ \\
F2 & $0.1 \mathrm{~g}$ & $0.1 \mathrm{~g}$ & $0.2 \mathrm{ml}$ \\
F3 & $0.1 \mathrm{~g}$ & $0.2 \mathrm{~g}$ & $0.1 \mathrm{ml}$ \\
F4 & $0.1 \mathrm{~g}$ & $0.2 \mathrm{~g}$ & $0.2 \mathrm{ml}$ \\
F5 & $0.1 \mathrm{~g}$ & $0.3 \mathrm{~g}$ & $0.3 \mathrm{ml}$ \\
F6 & $0.1 \mathrm{~g}$ & $0.3 \mathrm{~g}$ & $0.4 \mathrm{ml}$ \\
F7 & $0.1 \mathrm{~g}$ & $0.4 \mathrm{~g}$ & $0.5 \mathrm{ml}$ \\
F8 & $0.1 \mathrm{ml}$ & $0.4 \mathrm{~g}$ & $0.6 \mathrm{ml}$ \\
\hline
\end{tabular}

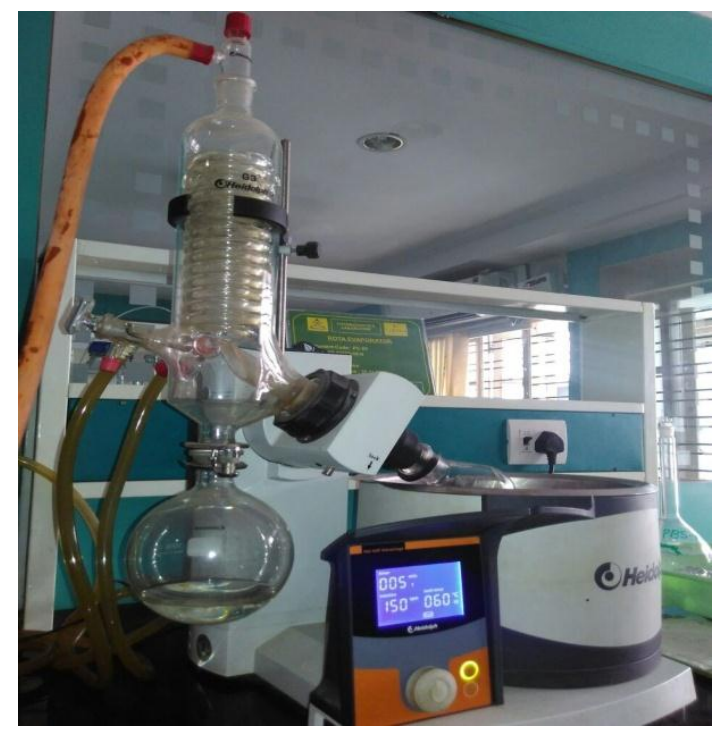

[A]

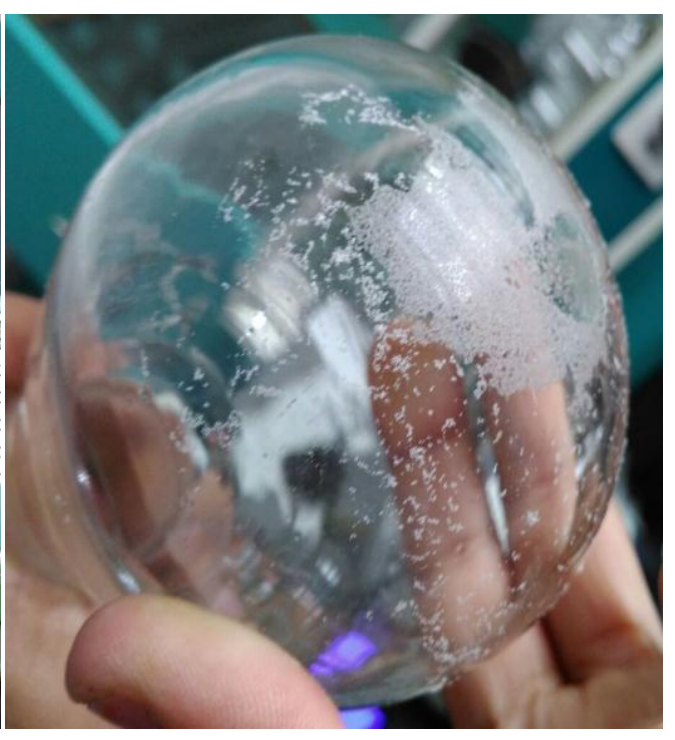

[B] 


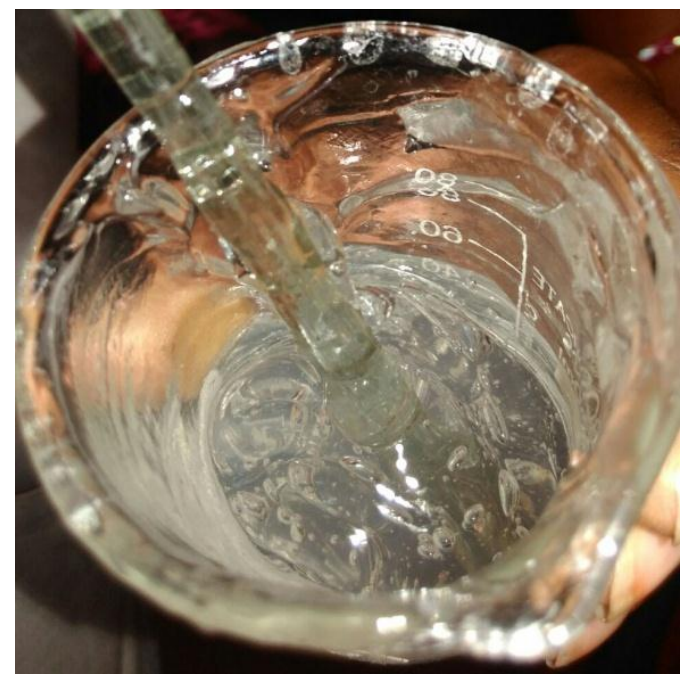

[C]

Figure-1: [A] Preparation of drug loadedniosomes using Rota evaporator [B] Thin film of niosomes. [C] Niosomal gel

\section{Formulation of Carbopol gel:}

1\% w/w Carbopol 934 was measured and sprinkled with continuous stirring into warm distilled water. This dispersion was hydrated for 1-2hours followed by the addition of Propylene Glycol $(10 \% \mathrm{w} / \mathrm{w})$ and Glycerol (30\% w/w) respectively with through continuous stirring.

The plain drug gel of Erythromycin 1\% was prepared by addition of drug to gel followed dispersion. The $\mathrm{pH}$ of the dispersion was adjusted to 6 using sodium hydroxide solution and the weight of the gel was attuned with distilled water. This gel formulation was sonicated for 30 minutes in bath sonicator Citizen, India CD4820 and left undisturbed overnight for removal of air bubbles.(Figure-1C)

The formulation of drug loaded niosomal gel was made by the addition of drug loaded niosomes in the similar manner as mentioned above with a quantity of equivalent to that of plain. $(8,9)$

\section{Characterization of niosomes:}

\section{Transmission Electron Microscope studies:}

Niosome vesicles were characterized by Transmision Electron Microscope studies. The TEM images of the niosomal formulation was made using JEOL JSM-6380 LA TEM. The TEM showed that niosomes vesicles were uniform in size and spherical.(10).

\section{FTIR Analytical studies:}

The FTIR analysis of the Erythromycin, Cholesterol, Carbopol gel and niosomal gel were carried out using FT-IR spectrophotometer of Bruker ALPHA-T-1020.

\section{Determination of $\mathbf{p H}$ :}


The digital pH meter (Lab India Sab $5000 \mathrm{pH}$ meter) was used for measurement of the $\mathrm{pH}$ of the niosomal gel. (11)

\section{Determination of entrapment efficiency percentage:}

The centrifugation method was used for estimation of the Erythromycin encapsulated in niosomes loaded in the carbopol gel. The niosomal formulation was collected after 3 minutes of sonication and subjected to centrifucation at $13000 \mathrm{rpm}$ for one and half an hour. After centrifugation $1 \mathrm{ml}$ of the supernatant was diluted with PBS-7.4.for UV analysis at 203.4nm. These studies revealed the total amount of free drug left over. The pellet formed after centrifugation contains the entrapped drug and which was added to the gel. Entrapment efficiency is expressed as the percent of drug trapped.(12,13)

$\%$ Entrapment $=$ Total drug - Diffused drug $/$ Total drug $\times 100$

\section{Percent Drug Content:}

$1 \mathrm{gm}$ of niosomal gel prepatation was collected and the niosomal vesicles were lysed using $25 \mathrm{ml}$ of methanol by sonication in bath sonicator Citizen, India CD4820 for 15 minutes. The dispersion was subjected to centrifugation at $14000 \mathrm{rpm}$ for 30 minutes. The supernatant was diluted to $100 \mathrm{ml}$ with methanol. From this $10 \mathrm{ml}$ solution was diluted to $100 \mathrm{ml}$ with phosphate buffered saline of $\mathrm{pH} 7.4$. The drug content was calculated using UV spectrophotometer at 203.4nm.

$\%$ Drug content $=$ Amount of Drug obtained after centrifugation/ Amount of drug taken $\times 100$

\section{$\%$ Drug entrapped and \% drug content:}

As the Formulations F1 to F4 has shown more entrapment than others they were analyzed for anti-microbial activity.

\section{Anti microbial activity:}

\section{Agar well diffusion assay}

The antibacterial activity of the extract was determined by agar well diffusion method. Briefly, overnight bacterial culture were diluted in the Mueller-Hinton broth to obtain a bacterial suspension of $108 \mathrm{CFU} / \mathrm{ml}$. Petri plates containing 20ml of Muller-Hinton broth Agar media were inoculated with $100 \mu \mathrm{l}$ of diluted cultures by spread plate technique and were allowed to dry in a sterile chamber. $5 \mathrm{~mm}$ well was cut using a cork borer on the surface of the inoculated agar. The gel was loaded into wells and was allowed to dry completely. The antibacterial activity was assessed by measuring the inhibition zone.

\section{Determination of MIC and Zone of Inhibition}


A minimum inhibitory concentration is the lowest concentration of an antimicrobial that inhibit the growth of micro-organism after 18-24hrs. The samples were tested at different concentration. Sterile NA plates were prepared and $0.1 \mathrm{ml}$ of the inoculums of test organism was spread uniformly. Wells were prepared by using a sterile borer of diameter $5 \mathrm{~mm}$ and niosomal gel of different formulations is added to the wells in separate petri plates aseptically. A same concentration of plain gel is also added to a separate well. A blank is prepared without adding any drug to one of the inoculated petri plate. The plates were incubated at $35-37 \mathrm{oC}$ for $18-48$ hours, a period of time sufficient for the growth. The zone of inhibition of microbial growth around the well was measured in $\mathrm{cm}$. MIC was calculated from the fully grown plates.

\section{Stability Studies of niosomes:}

After measuring the initial percentage entrapment of the drug in the optimized formulation, the three batches of the same formulation were stored in sealed glass ampoules (one each) at room temperature $\left(25+0.5^{\circ} \mathrm{C}\right)$ for a one month. The percentage entrapment of the drug and $\%$ drug content was determined in the formulations after 30 days to know the amount of drug leaked out. The percent drug lost was calculated taking the initial entrapment of drug as $100 \%$.

\section{RESULTS AND DISCUSSION:}

The surface morphology was studied by optical microscopy and transmission electron microscopy. The shapes of most of the erythromycin loaded niosomes were found spherical as shown in the following figures (Figure-2).

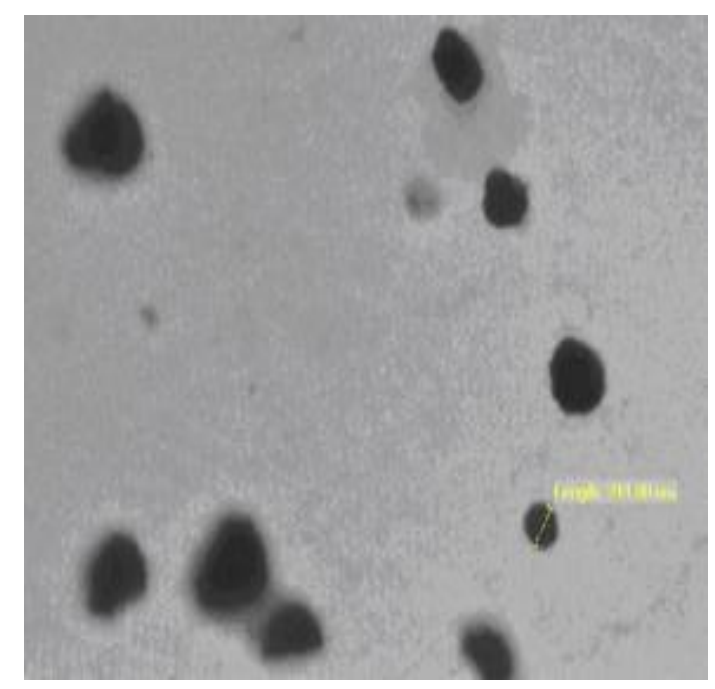

Figure-2 TEM Image of niosomes

The value of $\mathrm{pH}$ of topical niosomal gel was measured using digital $\mathrm{pH}$ meter at room temperature. The $\mathrm{pH}$ of all niosomal gels were found to be in the range of $7.4 \pm 0.02$ to $7.4 \pm 0.08$. 
Compatibility studies were performed using FTIR spectrophotometer. The IR Spectrum of pure Erythromycin and physical mixture of drug and other excipients were studied. The correlation between the pure drug and the excipients indicated that the drug was compatible with the formulation excipients. The FTIR studies reveal the non interaction between the drug and excipient. It was evident from infrared spectroscopy that the mixture of drug and exciepient was compatible because there were no alterations in band spectra when compared with each separate spectra of the drug. Two sharp bands were observed for erythromycin $(1,726.40 \mathrm{~cm}-1$ for $\mathrm{C}=\mathrm{O}$ str and 2,920.02 cm-1 for O-H str) (Figure-3) and two bands for cholesterol (1,056.61 cm-1 $\mathrm{C}=\mathrm{O}$ str ester, $1466.33 \mathrm{~cm}-1 \mathrm{C}-\mathrm{O}$ str, $3425.11 \mathrm{~cm}-1$, C-O str, )(Figure-4) two sharp bands for carbopol-934 (1,636.97 cm-1 C=O str ester, $3447.32 \mathrm{~cm}-1$, C-O str)(Figure-5) and optimized drug gel (1466.33 cm-1 C-O str, 1,738.62 cm-1 for C=O str, $2923.00 \mathrm{~cm}-1$ for O-H str, 3422.76 cm-1, C-O str),(Figure-6) (these are infrared spectroscopy band representative for functional group present in drugs, cholesterol and carbolpol gel ).

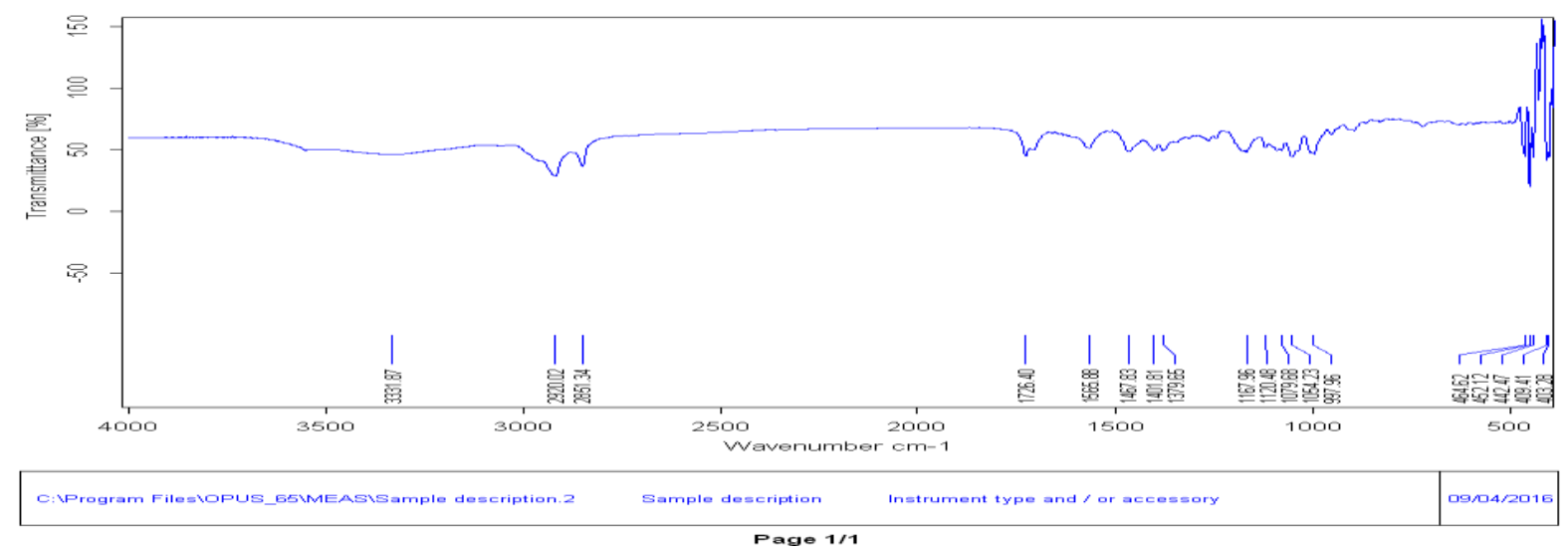

Figure-3 FTIR spectra of pure Erythromycin

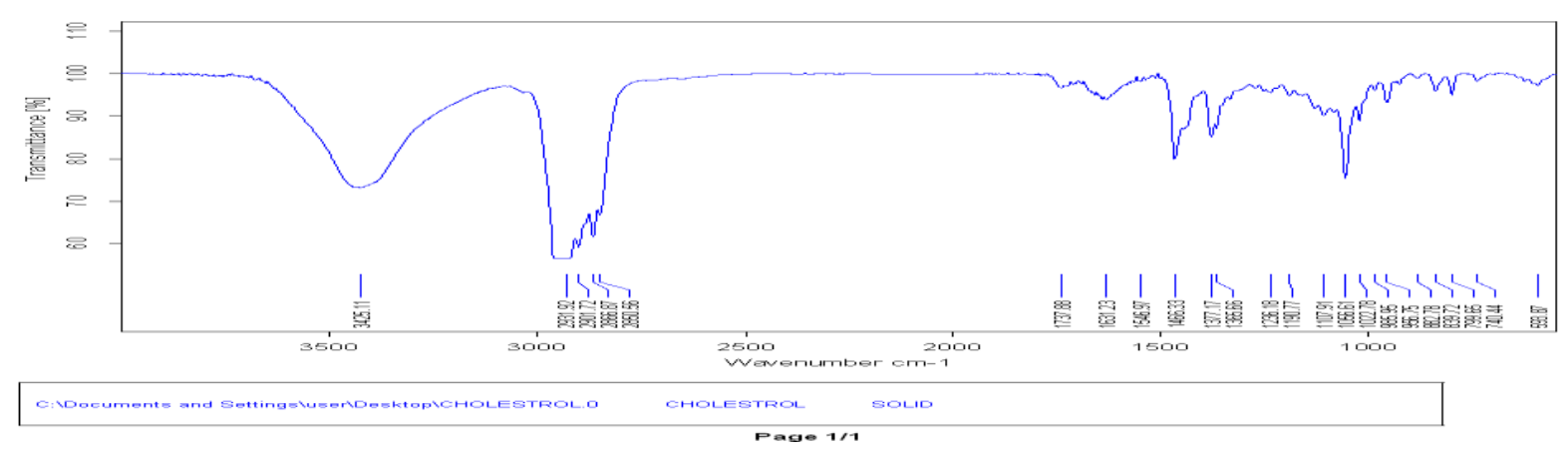

Figure-4 FTIR Spectra of Cholesterol 


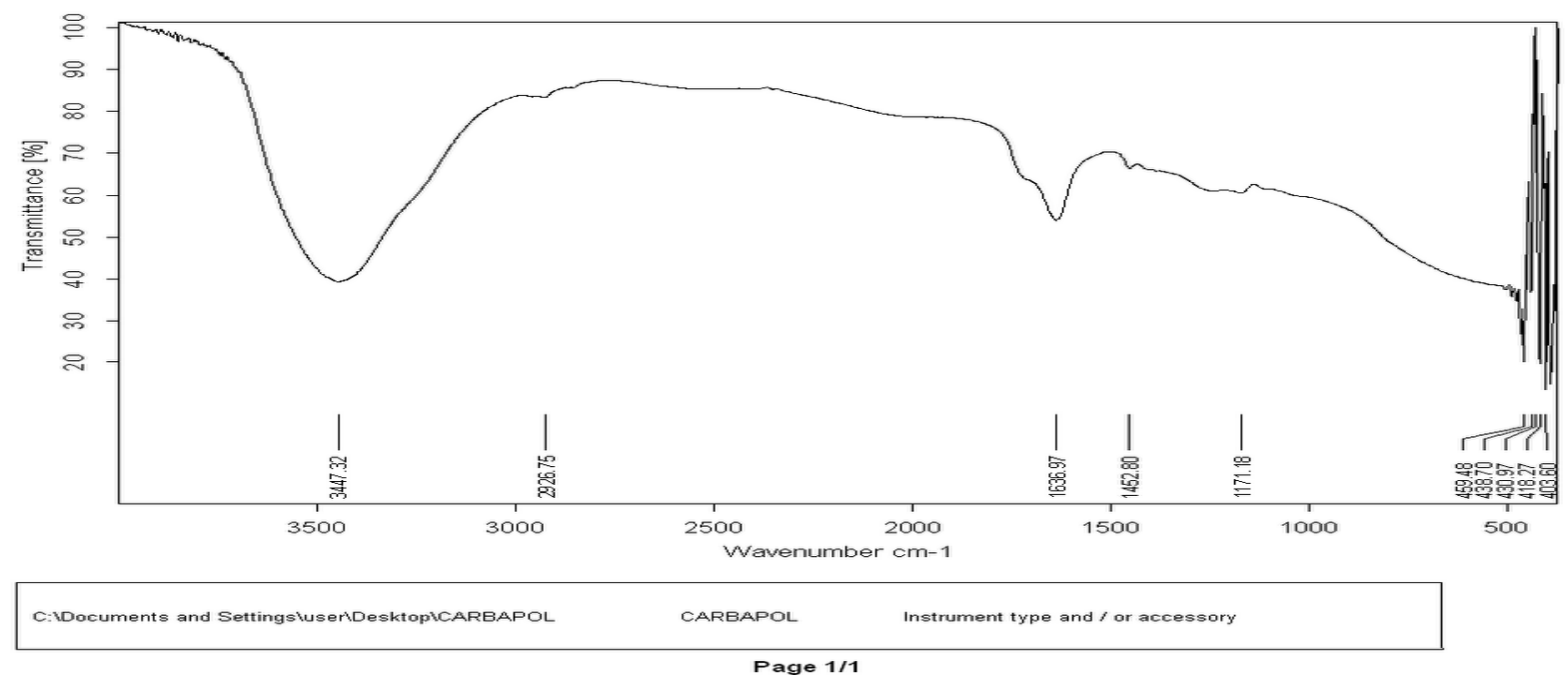

Figure-5 FTIR Spectra of Carbopol 934

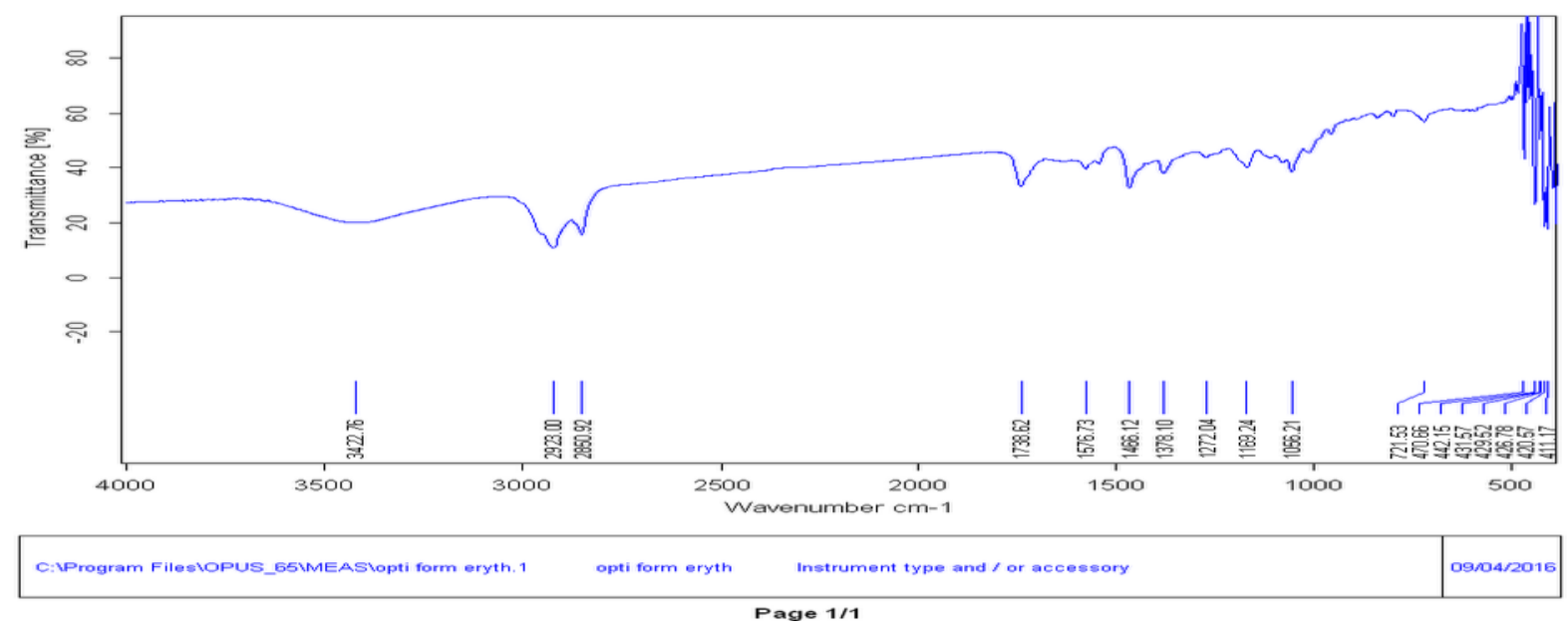

Figure-6 FTIR of optimized niosomal gel formulation

The percentage entrapment of erythromycin was found to be maximum with formulation F2 (maximum 93.4783). ( Table-2) These results can be explained by the fact that an increase in cholesterol content resulted in an increase of micro viscosity of the membrane indicating more rigidity of the bi layers. Cholesterol has the ability to cement the leaking space in the bi layer membranes. The entrapment efficiencies for niosomes prepared using span 80 directly influence the drug entrapment efficiency. The lower the HLB of the surfactant the higher will be the drug entrapment efficiency and stability as in the case of niosomes prepared using span 80 . The formulations F1 to F4 have shown more entrapment. Hence they were analyzed for antimicrobial activity.(Figure-7)

Table-2: \% Drug entrapped and \% drug content in niosomes

\begin{tabular}{lll}
\hline Formulation & \% Entrapment efficiency & \% Drug content \\
\hline F1 & 89.3287 & 95.3 \\
F2 & 93.4783 & 98.7 \\
\hline
\end{tabular}




\begin{tabular}{lll}
\hline F3 & 91.5678 & 95.6 \\
F4 & 88.9237 & 93.4 \\
F5 & 83.2592 & 92.4 \\
F6 & 86.5297 & 93.6 \\
F7 & 87.2235 & 90.5 \\
F8 & 81.0486 & 89.3 \\
\hline
\end{tabular}

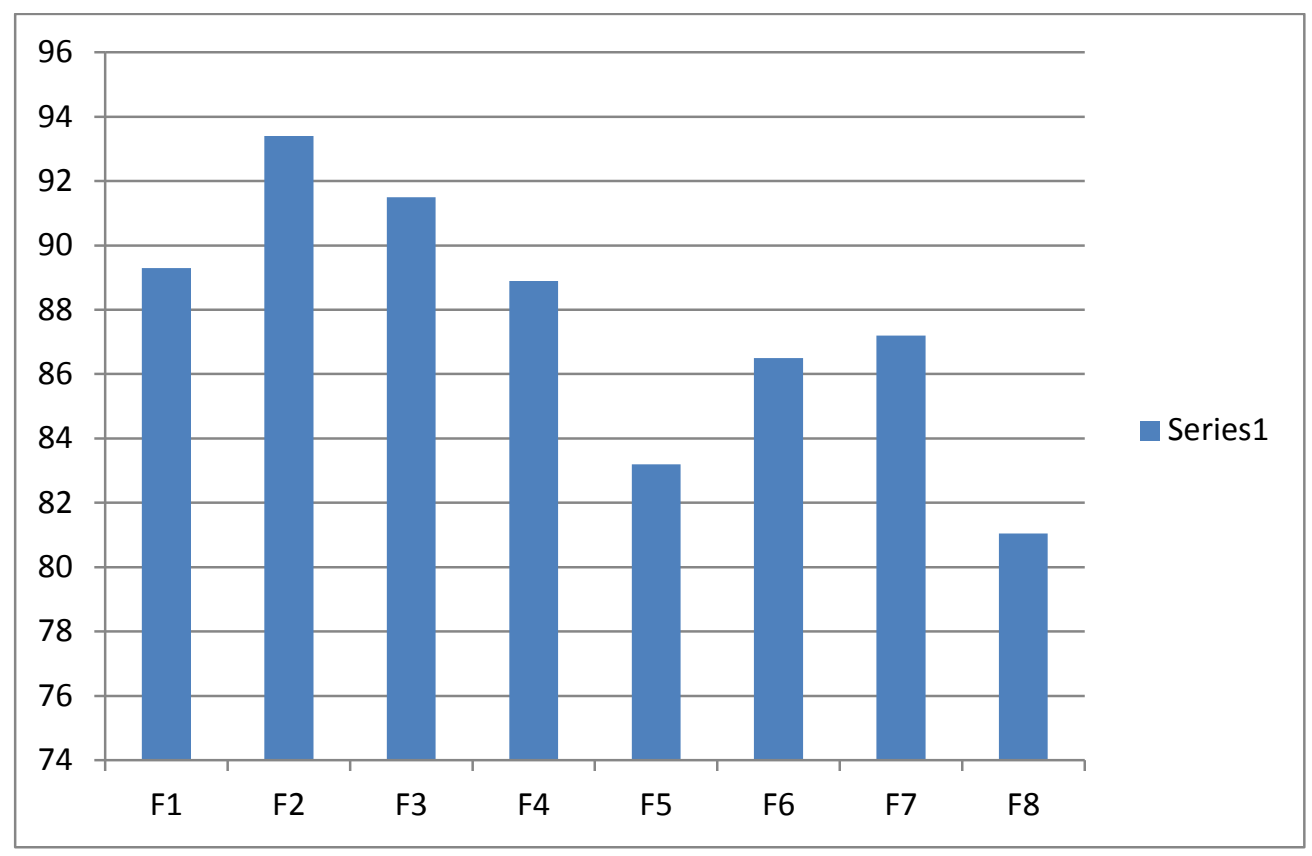

Figure-7 Entrapment efficiency of different formulations

The stability studies showed that niosomes have shown the minimum drug loss at $25 \pm 2{ }^{\circ} \mathrm{C}$ for period of 30 days respectively. The percentage entrapment efficiency and percentage drug content were comparatively good (Table-3).

\section{Anti-Microbial activity:}

From the results of anti-microbial activity, the zone of inhibition of niosomal gel is more than that of the plain gel. Further the drug from the plain gel was released at once but from the niosomal gel there was a slow release indicating that niosomal gel shows sustained release of erythromycin (Table-4). Out of all the formulations F2 has shown more zone of inhibition.(Figure-8,9) 


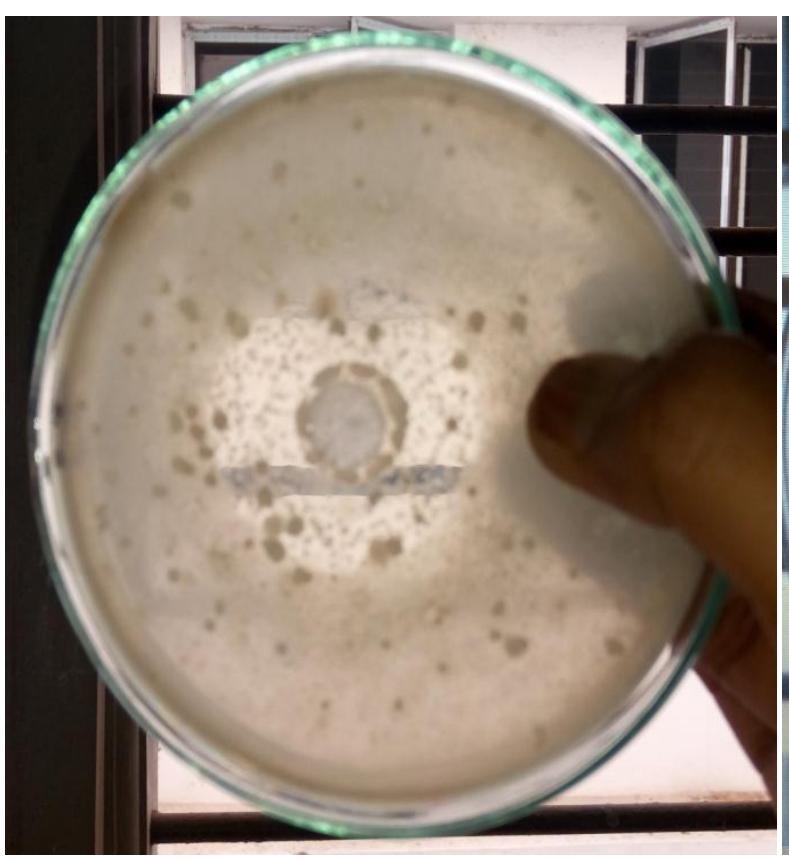

Figure-8 [A]

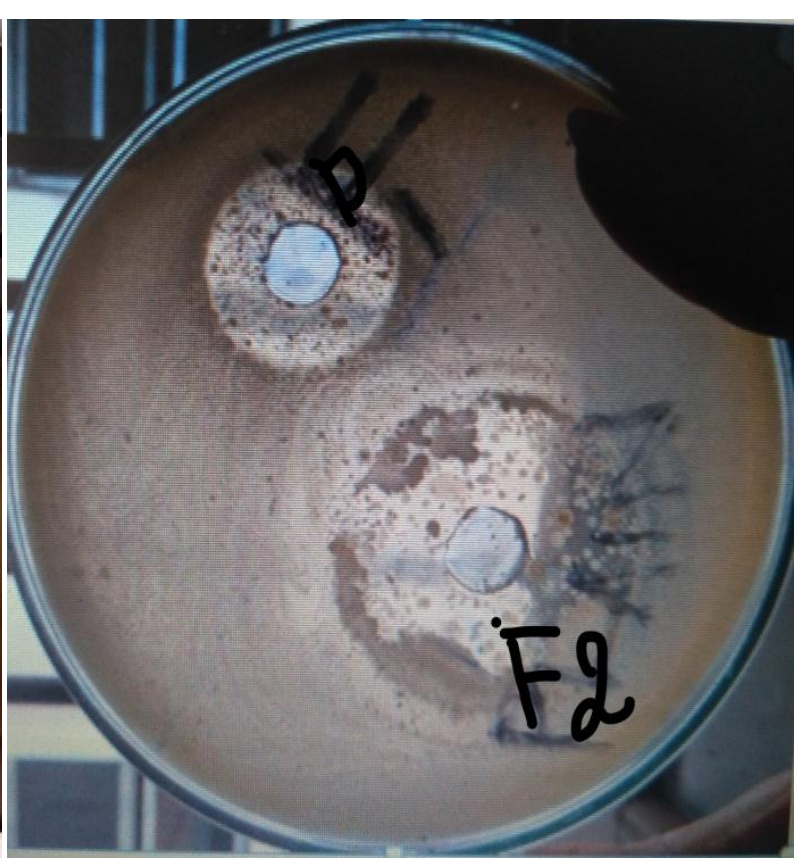

[B]

[A] Zone of inhibition of $\mathbf{F} 2$

[B] Zone of inhibition of plain gel and niosomal gel

Table -3:\% Entrapment efficiency and \% Drug content after stability studies.

\begin{tabular}{|c|c|c|c|c|}
\hline \multirow[t]{2}{*}{ Number of Days } & \multicolumn{2}{|c|}{$\begin{array}{l}\text { \% Entrapment Efficiency } \\
\text { at temperatures }\end{array}$} & \multicolumn{2}{|c|}{$\begin{array}{l}\text { \% Drug Content } \\
\text { at temperatures }\end{array}$} \\
\hline & Before $\left(25 \pm 2^{\circ} \mathrm{C}\right)$ & After $\left(25 \pm 2^{\circ} \mathrm{C}\right)$ & $\begin{array}{l}\text { Before } \\
\left(25 \pm 2^{\circ} \mathrm{C}\right)\end{array}$ & $\begin{array}{l}\text { After } \\
\left(25 \pm 2^{\circ} \mathrm{C}\right)\end{array}$ \\
\hline 30 & 91.87 & 89.66 & 92.04 & 91.26 \\
\hline
\end{tabular}

Table-4 Zone of inhibition of niosomal gel formulation and plain gel

\begin{tabular}{lll}
\hline Test organism & Formulation & Zone of Inhibition in mm \\
\hline Staphylococcus aureus MTCC 902 & F1 & 1.2 \\
Staphylococcus aureus MTCC 902 & F2 & 1.5 \\
Staphylococcus aureus MTCC 902 & F3 & 1.0 \\
Staphylococcus aureus MTCC 902 & F4 & 0.8 \\
Staphylococcus aureus MTCC 902 & Plain gel & 0.5 \\
\hline
\end{tabular}

\section{CONCLUSION:}

It can be concluded from the results of present study that niosomal gel improves the transdermal delivery, prolong the release, and improve the site specificity of the drug erythromycin. Niosomes formed from cholesterol: $\operatorname{span} 80(\% \mathrm{w} / \mathrm{w})$ is a promising approach to improve the permeability of erythromycin in a period of time. Niosomes creates a new opportunity for the well-controlled transdermal delivery of a number of drugs that have a problem of administration by other routes. 


\section{ACKNOWLEDGEMENT:}

Authors are thankful to Dr. P. Rajeshwar Reddy, Chairman and Dr.Vasudha Bakshi, Principal of Anurag group of Institutions (Formerly Lalitha College of Pharmacy), Venkatapur, Ghatkesar for providing the facilities for completion of this research work.

\section{REFERENCES:}

1. Betageri G. \& Habib M. Liposomes as drug carriers. Pharm. Eng. 1994:14 :76-77.

2. Schreier H. \& Bouwstra JA. Liposomes and niosomes as topical drug carriers: dermal and transdermal drug delivery. J. Con. Rel. 1994:30: 1-15.

3. Namedo A. \& Jain NK. Niosomes as drug carriers. Indian J. Pharm. Sci. 1996:58(2): 4146.

4. Junginger HE, Hofland HEJ \& Bouwstra JA. Liposomes and niosomes interaction with human skin. Cosmet. Toil. 1991:106: 45-50.

5. Azmin MN, Florence AT, Handjani-Vila RM, Stuart JFB, Vanlerberghe G \& Whittaker JS. The effect of non-ionic surfactant vesicle (niosome) entrapment on the absorption and distribution of methotrexate in mice. J. Pharm. Pharmacol. 1985:37: 237-242

6. Hofland HEJ, Vandergest R, Bodde HE, Junginger HE \& Bouwstra JA. Estradiol permeation from non ionic surfactant vesicles through human stratum corneum in-vitro. Pharm. Res. 1994:11: 659-664.

7. Vyas jigara*, Vyas pujab, Sawant krutikaa formulation and evaluation of topical niosomal gel of erythromycin Int J Pharm Pharm Sci, Vol 3, Issue1, 123126.

8. Friend DR. In vitro permeation techniques. J. Controlled Rel. 1992:18:235-248.

9. Tojo K. Design and calibration of in-vitro permeation apparatus. In: Transdermal Controlled Systemic Medications (Chien YW, Ed), Marcel Dekker, New York, 1987, 127-158.

10. Uchegdu IF \& Vyas SP. Non ionic surfactant based vesicles in drug delivery. Int. J. Pharmaceutics 1998:172: 33-70

11. Baillie AJ, Florence AT, Hume LR, Muirhead GT \& Rogerson A. The preparation and properties of non ionic surfactant vesicles. J. Pharm. Pharmcol. 1985:37: 863-868.

12. Bhaskaran S \& Panigrah L. Formulation and evaluation of niosomes using different non ionic surfactants. Ind. J. Pharm. Sci. 2002:1: 63-65. 
13. Jousma H, Joosten JGH \& Jumgimger HEJ. Mesophase in mixture of water and polyoxyethylene surfactant: Variations of repeat spacing with temperature and composition. Coll. Polyme. Sci. 1988:266(7): 640-651.

14. Ankush Gupta1,* Sima Singh1,* Niranjan GKotla1 Thomas J Webster2,3Formulation and evaluation of a topical niosomal gel containing a combination of benzoyl peroxide and tretinoin for antiacne activity International Journal of Nanomedicine 2015:10.

15. 15.Abhishek Budhiraja \& Garima Dhingra Development and characterization of a novel antiacne niosomal gel of rosmarinic acid Drug Deliv, 2015; 22(6): 723-730 